Author affiliations and support information (if applicable) appear at the end of this article.

Published at jco.org on February 2, 2018.

Processed as a Rapid Communication manuscript.

S.J.R. and M.A.S. contributed equally to this article.

Clinical trial information: NCT02181738.

Corresponding author: Margaret A. Shipp, MD, Dana-Farber Cancer Institute, 450 Brookline Ave, Mayer 513, Boston, MA 02215; e-mail: margaret_shipp@dfci. harvard.edu.

(C) 2018 by American Society of Clinical Oncology

0732-183X/18/3610w-942w/\$20.00

\title{
Major Histocompatibility Complex Class II and Programmed Death Ligand 1 Expression Predict Outcome After Programmed Death 1 Blockade in Classic Hodgkin Lymphoma
}

Margaretha G.M. Roemer, Robert A. Redd, Fathima Zumla Cader, Christine J. Pak, Sara Abdelrahman, Jing Ouyang, Stephanie Sasse, Anas Younes, Michelle Fanale, Armando Santoro, Pier Luigi Zinzani, John Timmerman, Graham P. Collins, Radhakrishnan Ramchandren, Jonathon B. Cohen, Jan Paul De Boer, John Kuruvilla, Kerry J. Savage, Marek Trneny, Stephen Ansell, Kazunobu Kato, Benedetto Farsaci, Anne Sumbul, Philippe Armand, Donna S. Neuberg, Geraldine S. Pinkus, Azra H. Ligon, Scott J. Rodig, and Margaret A. Shipp

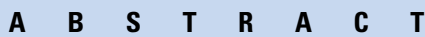

\section{Purpose}

Hodgkin Reed-Sternberg (HRS) cells evade antitumor immunity by multiple means, including gains of 9p24.1/CD274(PD-L1)/PDCD1LG2(PD-L2) and perturbed antigen presentation. Programmed death 1 (PD-1) receptor blockade is active in classic Hodgkin lymphoma (cHL) despite reported deficiencies of major histocompatibility complex (MHC) class I expression on HRS cells. Herein, we assess bases of sensitivity to PD-1 blockade in patients with relapsed/refractory cHL who were treated with nivolumab (anti-PD-1) in the CheckMate 205 trial.

\section{Methods}

HRS cells from archival tumor biopsies were evaluated for 9p24.1 alterations by fluorescence in situ hybridization and for expression of PD ligand 1 (PD-L1) and the antigen presentation pathway components- $\beta 2$-microglobulin, $\mathrm{MHC}$ class I, and $\mathrm{MHC}$ class II-by immunohistochemistry. These parameters were correlated with clinical responses and progression-free survival (PFS) after PD-1 blockade.

\section{Results}

Patients with higher-level 9p24.1 copy gain and increased PD-L1 expression on HRS cells had superior PFS. HRS cell expression of $\beta 2$-microglobulin/MHC class I was not predictive for complete remission or PFS after nivolumab therapy. In contrast, HRS cell expression of MHC class II was predictive for complete remission. In patients with a $>12$-month interval between myeloablative autologous stem-cell transplantation and nivolumab therapy, HRS cell expression of MHC class II was associated with prolonged PFS.

\section{Conclusion}

Genetically driven PD-L1 expression and MHC class II positivity on HRS cells are potential predictors of favorable outcome after PD-1 blockade. In cHL, clinical responses to nivolumab were not dependent on HRS cell expression of MHC class I.

\section{J Clin Oncol 36:942-950. (O) 2018 by American Society of Clinical Oncology}

\section{INTRODUCTION}

Classic Hodgkin lymphomas (cHLs) are composed of rare malignant Hodgkin Reed-Sternberg (HRS) cells within an extensive inflammatory and immune cell infiltrate. ${ }^{1}$ Despite this $\mathrm{T}$ cell-rich infiltrate, HRS cells evade effective antitumor immune responses by multiple mechanisms. ${ }^{1-4}$ HRS cells exhibit frequent copy number alterations of 9p24.1 and the genes encoding the programmed death 1 (PD-1) receptor ligands, $P D-L 1$ and $P D-L 2$ (also called CD274 and PDCD1LG2, respectively), ranging from low-level polysomy to relative copy gain and high-level amplification and copy numberdependent increased expression of PD-1 ligands. ${ }^{3,4}$ PD-1 ligands engage the PD-1 receptor on T cells, inhibiting T-cell activation and antitumor immune responses. ${ }^{5}$

The identified 9p24.1 alterations and PD-1 ligand overexpression in cHL prompted clinical evaluation of PD-1 blockade in patients with 
relapsed/refractory disease. In pilot and registration trials of the PD-1-blocking antibody nivolumab, patients with relapsed/refractory $\mathrm{cHL}$ and limited treatment options had response rates of $65 \%$ to $87 \%$ and prolonged progression-free survival (PFS). ${ }^{6,7}$ Similar results were obtained with a second PD-1-blocking antibody, pembrolizumab. ${ }^{8,9}$ These findings - the highest reported response rates to PD-1 blockade in any tumor type-led to rapid approval by the US Food and Drug Administration and the European Medicines Agency of both PD-1 antibodies for the treatment of relapsed/refractory cHL.

In murine cancer models and human solid tumors, the efficacy of PD-1 blockade has been attributed to activation of CD8 ${ }^{+}$ cytotoxic T cells in the tumor microenvironment. ${ }^{10-13} \mathrm{CD}^{+} \mathrm{T}$ cells require antigen presentation via major histocompatibility complex (MHC) class I molecules that are transported to the cell surface in association with $\beta 2$-microglobulin $(\beta 2 \mathrm{M})$. However, HRS cells exhibit frequent inactivating mutations and copy loss of $B 2 M$, leading to decreased or absent cell surface expression of MHC class I. ${ }^{14,15}$

An alternate mechanism of antitumor immunity depends on MHC class II-mediated antigen presentation to $\mathrm{CD} 4^{+}$effector cells. ${ }^{16-18}$ Unlike solid tumors, which are largely MHC class II negative, HRS cells frequently express MHC class II, likely because of their derivation from germinal center B cells. However, a subset of patients with cHL have genetic bases for deficient MHC class II expression and decreased/absent cell surface MHC class II on HRS cells. ${ }^{15,19-21}$ In recent imaging analyses of the intact cHL tumor microenvironment, we found that $\mathrm{PD}-\mathrm{Ll}^{+} \mathrm{HRS}$ cells were significantly more likely to be in physical contact with PD- $1^{+} \mathrm{CD} 4^{+}$ $\mathrm{T}$ cells than PD- $1^{+} \mathrm{CD}^{+} \mathrm{T}$ cells. $^{22}$

In this study, we assessed the possible predictive value of 9p24.1/PD-L1/PD-L2 genetic alterations and PD-L1 expression for clinical outcome in patients with relapsed/refractory cHL who received nivolumab in the CheckMate 205 trial. We also evaluated HRS cell expression of $\beta 2 \mathrm{M}, \mathrm{MHC}$ class I, and MHC class II and the association of these antigen presentation proteins with outcome after nivolumab therapy.

\section{METHODS}

\section{Clinical Data}

CheckMate 205 is a multicenter, multicohort, phase II trial of singleagent nivolumab in patients with cHL (ClinicalTrials.gov identifier: NCT02181738). ${ }^{7}$ The research protocol was approved by the respective institutional review boards; all participants gave written informed consent. The current biomarker study focused on cohorts B and C: patients with relapsed/refractory disease who previously underwent autologous stemcell transplantation (ASCT) and received brentuximab vedotin (BV) before and/or after ASCT. Patients were treated with nivolumab $3 \mathrm{mg} / \mathrm{kg}$ every 2 weeks until disease progression or unacceptable toxicity. Best overall response (BOR) and PFS were assessed by an independent review committee (IRC) using 2007 International Working Group response criteria. $^{23}$

\section{Fluorescence In Situ Hybridization}

In patients with available archival tumor biopsies, 9p24.1 genetic alterations were evaluated by fluorescence in situ hybridization (FISH) assay; probes encompassed CD274 (PD-L1, red) or PDCD1LG2 (PD-L2, green) and included a centromeric control (aqua). Copy number alterations were defined as previously described ${ }^{4}$ on the basis of the target: control signal ratio. Fifty HRS cells per tumor were analyzed. Nuclei with a target:control signal ratio of $\geq 3: 1$ were defined as coamplified for $P D-L 1$ and $P D-L 2$, and those with a signal ratio of $>1: 1$ but $<3: 1$ were classified as having relative copy gain of these loci. Nuclei with a signal ratio of $1: 1$, but more than two copies per probe, were defined as polysomic for 9p24.1. For each patient, the percentage and magnitude of 9p24.1 amplification, copy gain, polysomy, and normal copy numbers (disomy) were noted. Patients were classified by the highest observed level of 9p24.1 genetic alteration; those with 9p24.1 copy gain lacked amplification, and those with $9 \mathrm{p}$ polysomy lacked $9 \mathrm{p} 24.1$ copy gain or amplification.

\section{Immunohistochemistry}

Dual immunohistochemical staining of PD-L1 (clone 405.9A11 ${ }^{24}$ ) and PAX5 (24/Pax-5; BD Biosciences, San Jose, CA) was performed to delineate PD-L1 expression in PAX5 ${ }^{\mathrm{dim}+}$ malignant HRS cells on archival tumor biopsies, as previously described. ${ }^{4}$ A modified PD-L1 H-score (range, 0 to 300) was calculated by multiplying the percentage of PAX $5^{\mathrm{dim}+}$ (malignant) cells with positive PD-L1 staining ( $0 \%$ to $100 \%)$ and the average intensity of staining ( 0 to $3+$ on 50 HRS cells). Dual immunohistochemical staining of the antigen presentation components $\beta 2 \mathrm{M}$ (A0072, 1:6,000; Dako, Troy, MI), MHC class I (EMR8-5, 1:6,000; Abcam, Cambridge, MA), and MHC class II (CR3/43 M0775, 1:750; Dako) with PAX5 (BD Biosciences) was performed using an automated staining system (Bond III; Leica Biosystems, Vista, CA) according to the manufacturer's protocol.

Scoring criteria for samples in this trial followed the three-tiered scoring system previously established in the analysis of newly diagnosed cHLs treated with standard induction therapy. ${ }^{15}$ Briefly, two expert hematopathologists (S.J.R. and G.S.P.) independently reviewed each case. After their initial assessment, the two pathologists reviewed each case together and recorded a single consensus score. ${ }^{15}$ Each tumor was classified as positive, decreased, or negative for expression on HRS cells, relative to staining on adjacent nonmalignant inflammatory cells. In each case, areas with adjacent HRS cells were closely analyzed for HRS cell membrane expression of $\beta 2 \mathrm{M}, \mathrm{MHC}$ class I, and MHC class II, a process analogous to that used for the clinical evaluation of CD45 (leukocyte common antigen). For cases categorized as positive, at least $90 \%$ of evaluable HRS cells showed positive membrane staining for the biomarker at levels equivalent to, or greater than, that of adjacent nonmalignant inflammatory cells. ${ }^{15}$ For cases categorized as negative, at least $90 \%$ of evaluable HRS cells showed no detectable membrane staining for the biomarker relative to nonmalignant inflammatory cells. ${ }^{15}$ For cases categorized as decreased, positive membrane staining of HRS cells was present and unequivocally reduced relative to surrounding cells, and/or positive staining was observed in $<90 \%$ of evaluable HRS cells. Stained slides were scored blinded to the outcome data.

\section{Statistical Analyses}

IRC assessment of BOR was obtained in all treated patients at the database lock in December 2016. BOR was defined as the best response (complete remission [CR], partial remission [PR], stable disease, progressive disease $[\mathrm{PD}]$, or nonevaluable disease) between the first study treatment date and the first progression date or first subsequent therapy date, whichever occurred first. The modified H-score for PD-L1 expression was divided into four quartiles. Exact Kruskal-Wallis and JonckheereTerpstra tests were used to analyze singly and doubly ordered categorical associations, respectively. IRC-assessed PFS was defined as time from the date of first treatment until the date of progression or death. Patients who underwent subsequent anticancer treatment without reported progression were censored at the last tumor assessment before the additional anticancer therapy. Time-to-event analyses were performed using the Kaplan-Meier method, and SEs were calculated by Greenwood's formula. Differences in PFS curves were assessed with log-rank tests. Kruskal-Wallis and Jonckheere-Terpstra tests were performed with StatXact software (Cytel, Cambridge, MA); additional analyses were performed with $\mathrm{R}$ version 3.2.2 (R Foundation). All $P$ values were two-sided and nominal. 


\section{RESULTS}

\section{Patient Characteristics}

A total of 180 patients with relapsed/refractory $\mathrm{cHL}$ were treated in cohorts B and C of CheckMate 205. All 80 patients in cohort $\mathrm{B}$ received single-agent $\mathrm{BV}$ after relapsing from ASCT $(\mathrm{ASCT} \rightarrow \mathrm{BV})$. In contrast, cohort $\mathrm{C}(\mathrm{n}=100)$ included patients who received BV before ASCT for treatment of relapse $(\mathrm{BV} \rightarrow \mathrm{ASCT}$, $\mathrm{n}=33$ ), BV after relapse from ASCT (ASCT $\rightarrow$ BV, $n=58$ ), or BV before and after relapse from ASCT $(B V \rightarrow A S C T \rightarrow B V, n=9$; Data Supplement Table A1A and Fig A1).

An identified clinical difference in patients from cohorts $B$ and $\mathrm{C}$ was the time (median [range]) between prior myeloablative ASCT and nivolumab therapy (cohort B, 40 [2 to 228] months, versus cohort C, 21 [3 to 204] months; $P<.001$; Data Supplement Table A1A). This difference largely reflected the sequence of prior therapies for relapsed disease in the respective cohorts. In the cohort $\mathrm{B}$ ASCT $\rightarrow$ BV and cohort $\mathrm{C} \mathrm{ASCT} \rightarrow \mathrm{BV}$ and $\mathrm{BV} \rightarrow \mathrm{ASCT} \rightarrow \mathrm{BV}$ subsets, time intervals between myeloablative ASCT and nivolumab therapy were 40 months ( 2 to 228 months), 32 months ( 3 to 204 months), and 23 months (7 to 40 months), respectively. In the cohort C BV $\rightarrow$ ASCT subset, the time interval between ASCTand treatment was only 10 months ( 3 to 42 months) months ( $P<.001$; Data Supplement Table A1A).

We postulated that patients with a shorter interval between myeloablative ASCT and nivolumab therapy were still actively reconstituting their immune repertoire and tumor microenvironment at study entry. ${ }^{25-27}$ For this reason, we analyzed patients who were treated with nivolumab $\leq 12$ months and $>12$ months after ASCT separately in these exploratory analyses, in addition to evaluating all patients in cohorts B and C. The available biomarker data and clinical responses for patients are summarized in the Data Supplement Table A1B and Fig A1. Patients with evaluable biopsy specimens had PFS rates that were comparable to those of all treated patients in cohorts B and C (Data Supplement Fig A2).

\section{9p24.1 Genetic Alterations and PD-L1 Expression}

In total, 99 of 180 patients had evaluable tumor biopsy specimens for 9p24.1 FISH (Data Supplement Table A1B); all 99 had detectable 9p24.1 alterations in HRS cells: polysomy in 10 of $99(10 \%)$, copy gain in 59 of $99(60 \%)$, and amplification in 27 of 99 (27\%; Fig 1A). Patients were classified by the highest-level 9p24.1 alterations (Figs 1A and 1B), as previously described. ${ }^{4}$ Tumors with 9p24.1 amplification had additional HRS cells with copy gain (6\% to $84 \%$ ), polysomy ( $4 \%$ to $44 \%$ ), and disomy ( $2 \%$ to $45 \%$ ); those with relative copy gain had additional cells with polysomy (2\% to $84 \%$ ) and disomy ( $4 \%$ to $86 \%$ ), and those categorized as polysomic had additional residual disomic HRS cells (20\% to 92\%; Fig 1B). The percentage of residual disomic cells was lowest in tumors with amplification, intermediate in tumors with copy gain, and highest in tumors with polysomy $(P<.001$; Fig $1 C)$, consistent with an ordered spectrum of 9 p24.1 genetic alterations in $\mathrm{cHL}$.

Of patients with available 9p24.1 genetic data, 97 of 99 could be analyzed for PD-L1 protein expression (Data Supplement Table A1B). There was a significant association between PD-L1 protein expression (H-score) and the magnitude of 9p24.1 copy number alterations in HRS cells $(P=.001$; Fig 1D). Similar results were obtained for patients with intervals of $\leq 12$ or $>12$ months between ASCT and nivolumab therapy (Data Supplement Fig A3).
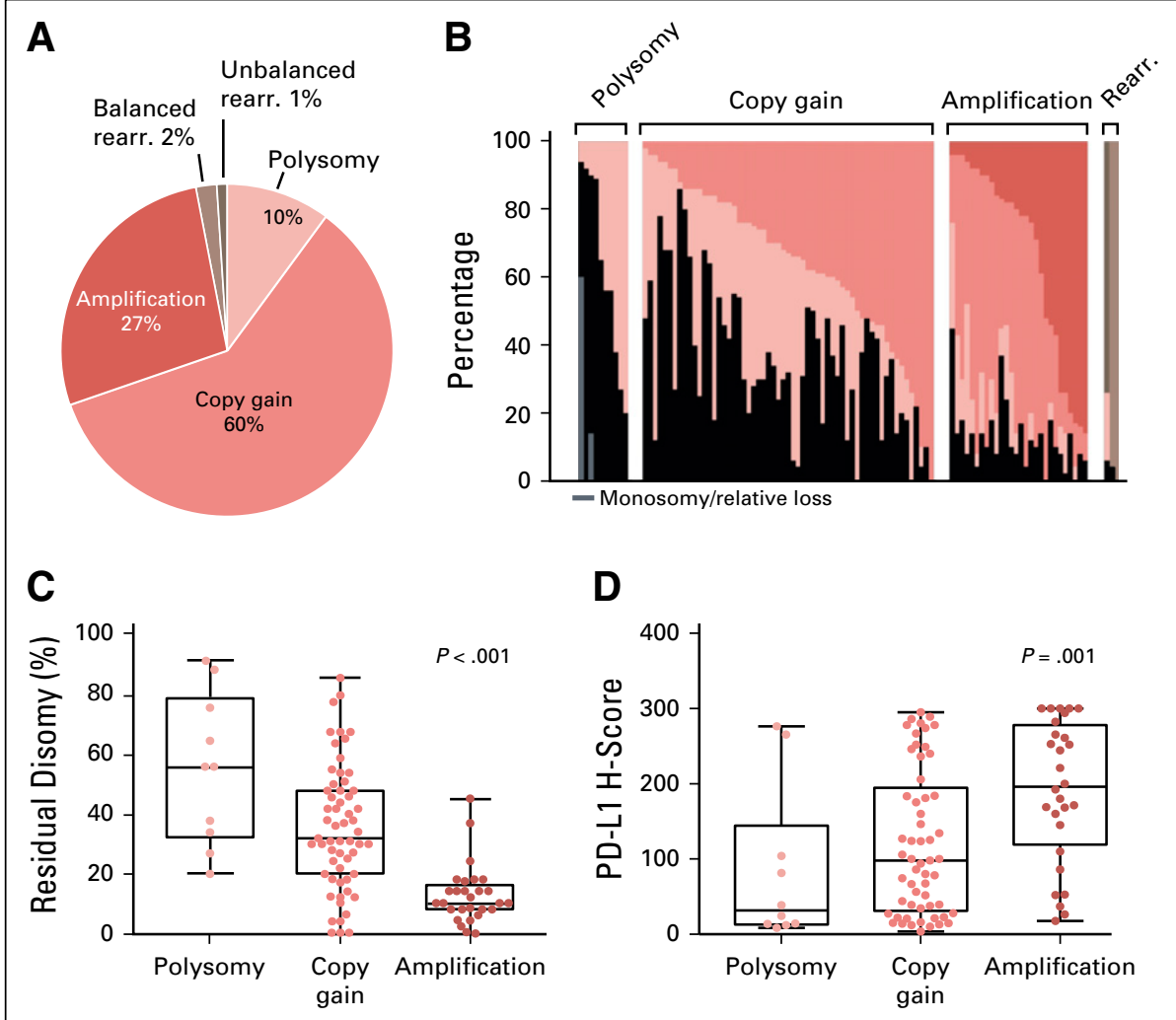

Fig 1. Prevalence of 9p24.1 genetic alterations and association of residual disomy and programmed death ligand 1 (PD-L1) H-scores with 9p24.1 genetic categories. (A) Prevalence of 9p24.1 genetic alterations in classic Hodgkin lymphomas (cHLs). (B) The spectrum of 9p24.1 alterations in evaluated cHLs. Each patient is classified by the highest observed level of 9p24.1 alteration in Hodgkin Reed-Sternberg (HRS) cells: polysomy, copy gain, amplification, or rearrangement (rearr., top). Individual patients are visualized as columns on the $x$-axis, and the percentage of HRS cells with monosomy/relative loss (gray), disomy (black), polysomy (light pink), copy gain (medium pink), amplification (red), balanced rearrangement (taupe), and/or unbalanced rearrangement (dark brown) is depicted on the $y$-axis. (C) Percentage of HRS cells with residual 9p24.1 disomy in cHLs classified by 9 p24.1 genetic categories $(P<.001$, Kruskal-Wallis rank sum test). (D) PD-L1 H-scores in CHLs classified by 9p24.1 genetic categories $(P=.001$, Kruskal-Wallis rank sum test). Ninety-nine patients were evaluable for $9 p 24.1$ alterations. Two patients had missing PD-L1 H-scores; 97 patients were evaluable for PD-L1 expression. 
In addition to copy number changes, chromosomal rearrangements of 9p24.1 were observed in three of $99(3 \%)$ patients (Figs $1 \mathrm{~A}$ and $1 \mathrm{~B})$. Two (2\%) patients had balanced rearrangements by FISH, positive PD-L2 expression, and no PD-L1 immunostaining (Data Supplement Fig A4; patients 1 and 2), indicative of $P D-L 2$ translocations. The third patient $(1 \%)$ had an unbalanced rearrangement, with extra copies of $P D-L 1$ and selective membranous PD-L1 expression (Data Supplement Fig A4; patient 3).

\section{BOR and PFS According to 9p24.1 Alterations and PD-L $1 \mathrm{H}$-Scores}

We next evaluated the potential associations between BOR and defined 9p24.1 alterations and PD-L1 H-scores (Figs 2A and $2 \mathrm{~B})$. The majority of evaluable patients responded to PD-1 blockade (Figs $2 \mathrm{~A}$ and $2 \mathrm{~B}$ ). In contrast to patients who obtained CR or PR or had stable disease, those who experienced progression on therapy were more likely to have lower-level 9p24.1 alterations (polysomy; Fig 2A and Data Supplement Fig A5A; PD $v$ non-PD, $P=.006$ ) and less PD-L1 expression on HRS cells (Fig 2B and Data Supplement Fig A5B; PD $v$ non-PD, $P=.047)$. Patients whose tumors had lower-level 9p24.1 alterations and less PD-L1 expression on HRS cells also had shorter PFS (Fig 2C, $P<.001$, and Fig $2 \mathrm{D}, P=.026$ ).

A patient whose tumor had an unbalanced rearrangement involving $P D-L 1$ obtained a PR and had prolonged PFS (patient 3, 14.8+ months; Data Supplement Fig A4A). The two patients with balanced $P D-L 2$ rearranged tumors had rapidly PD (PFS was 1.4 months in patient 1 and 2.1 months in patient 2; Fig $2 \mathrm{C}$ and Data Supplement Fig A4A).

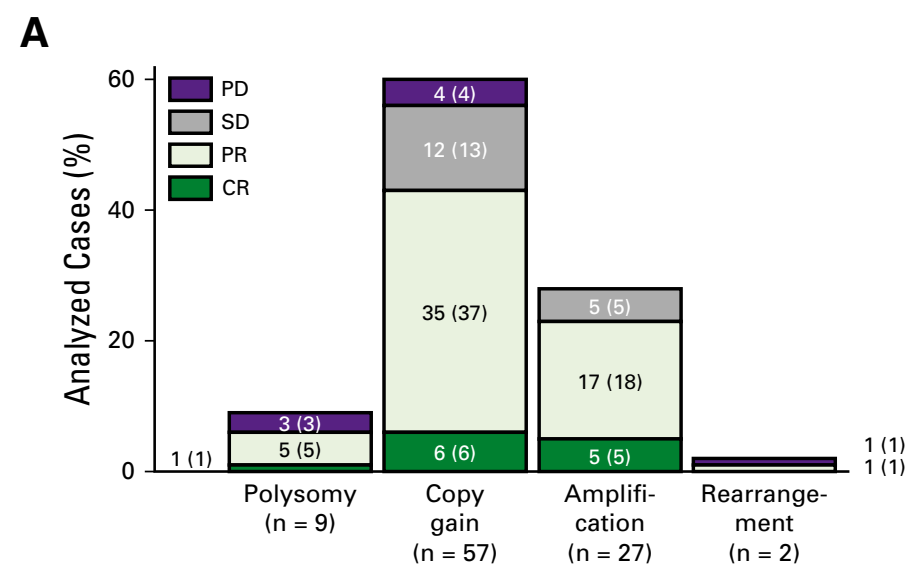

C

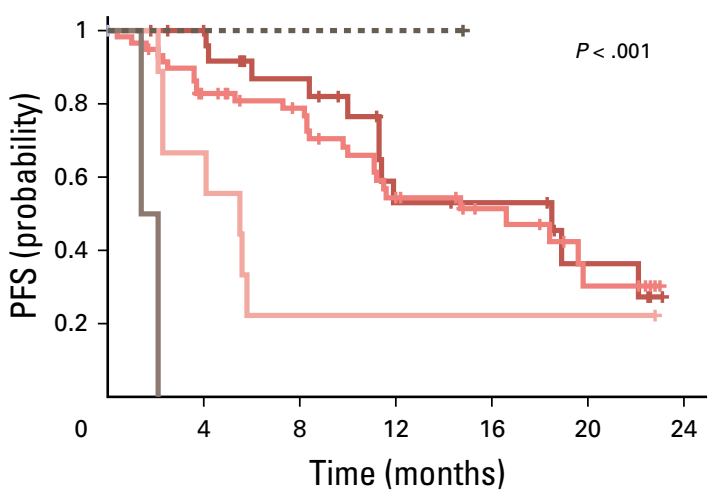

No. at risk

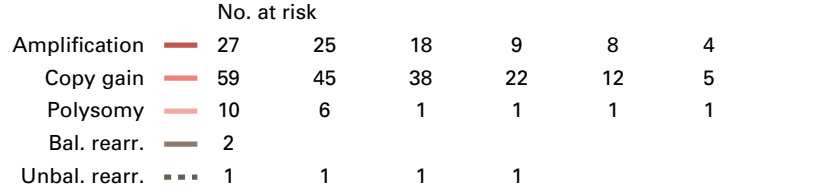

B

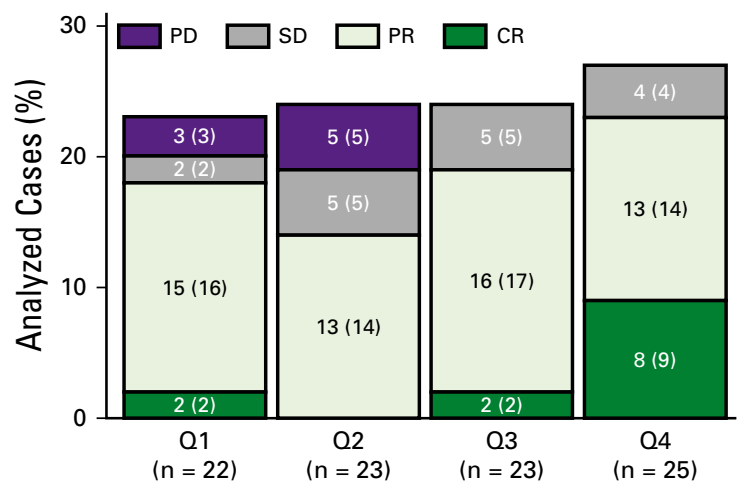

D

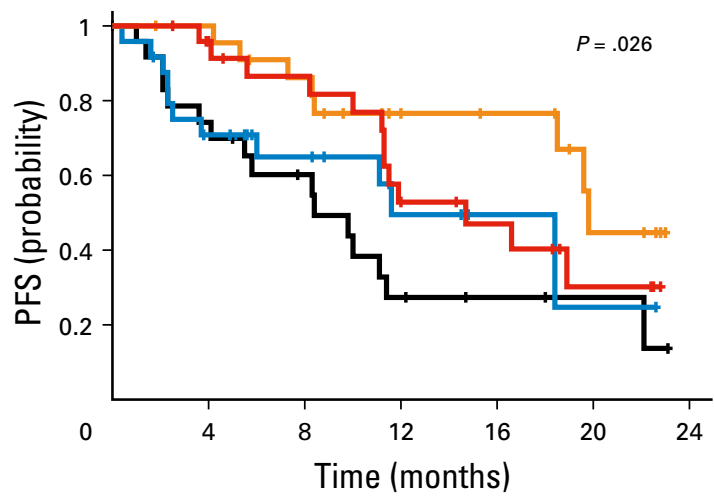

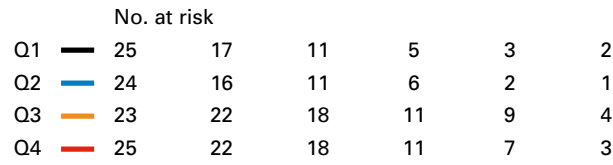

Fig 2. Best overall response (BOR) and progression-free survival (PFS) by 9p24.1 genetic category and programmed death ligand 1 (PD-L1) $H$-Scores. Ninety-nine patients

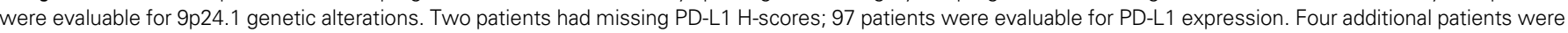

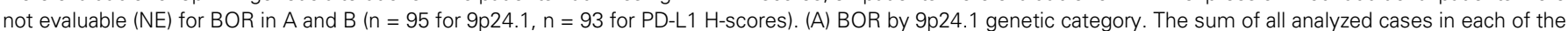

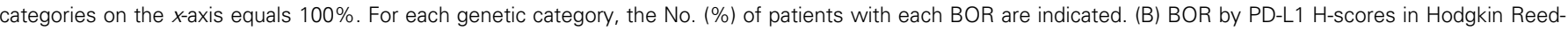

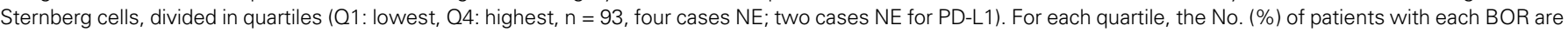

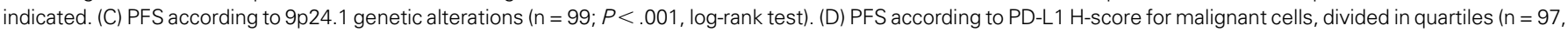

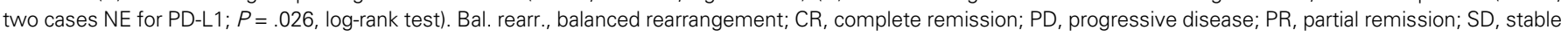
disease; Unbal. rearr., unbalanced rearrangement. 


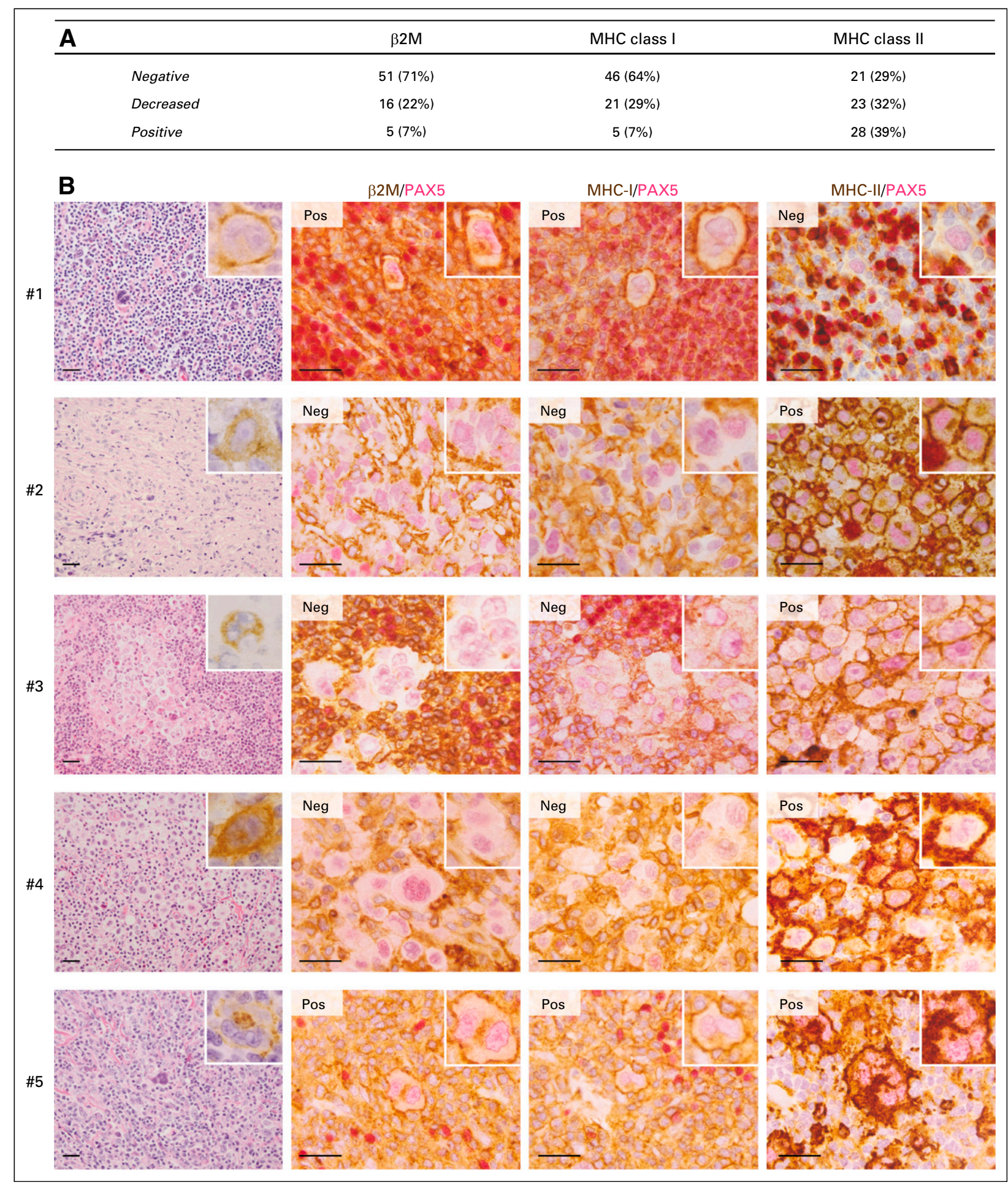

Fig 3. $\beta 2$-microglobulin ( $\beta 2 \mathrm{M})$, major histocompatibility complex ( $\mathrm{MHC}$ ) class I and $\mathrm{MHC}$ class II expression. (A) $\beta 2 \mathrm{M}$, $\mathrm{MHC}$ class I, and $\mathrm{MHC}$ class II expression in patients in whom all three antigen presentation components were evaluable $(n=72)$. (B) Representative images from five trial patients. Images include: a low-power $(\times 400)$ hematoxylin and eosin stain (left panels) with a CD30+ Hodgkin Reed-Sternberg (HRS) cell in the inset (right upper corner); and high-power ( $\times 1,000) \beta 2 \mathrm{M} / \mathrm{PAX} 5$, MHC class I (MHC-I)/PAX5, and MHC class II (MHC-II)/ PAX5 double stains (middle and right panels) with representative HRS cells in insets (right upper corner). For cases categorized as positive (Pos), at least $90 \%$ of evaluable PAX ${ }^{\text {dim+ }}$ HRS cells showed positive membrane staining for $\beta 2 \mathrm{M}, \mathrm{MHC}$ class I, and/or $\mathrm{MHC}$ class II at levels equivalent to or greater than that of adjacent nonmalignant inflammatory cells. In negative cases (Neg), at least $90 \%$ of evaluable PAX5 ${ }^{\text {dim+ }}$ HRS cells showed no detectable membrane staining for the biomarkers relative to adjacent nonmalignant inflammatory cells. HRS cells from patient 1 were positive (Pos) for $32 \mathrm{M}$ and MHC class I and negative (Neg) for MHC class II. This patient had stable disease as best overall response. HRS cells from patients 2, 3, and 4 were negative for $\beta 2 \mathrm{M}$ and $\mathrm{MHC}$ class I and positive for MHC class II. All three patients obtained complete remissions. HRS cells from patient 5 were positive for $\beta 2 \mathrm{M}, \mathrm{MHC}$ class I, and MHC class II. This patient achieved a partial remission. Magnification $\times 1,000$. Scale bar, $50 \mu \mathrm{m}$. 
In patients with intervals of $\leq 12$ or $>12$ months between ASCT and nivolumab therapy, the magnitude of 9p24.1 alterations was significantly associated with PFS (Data Supplement Fig A5C-D). The levels of PD-L1 protein expression were significantly associated with PFS in patients with $>12$ months between ASCT and nivolumab therapy (Data Supplement Fig A5F) but not in those with $\mathrm{a} \leq 12$-month interval (Data Supplement Fig A5E). In the latter group of patients, who were still reconstituting their immune repertoire and tumor microenvironment after myeloablative ASCT, PD-L1 expression may have been driven by additional microenvironmental signals that would not be reflected in the analyzed archival tumor biopsies (Data Supplement Fig A5E).

\section{Antigen Presentation in Patients Treated With Nivolumab}

Given the importance of effective antigen presentation for $\mathrm{T}$-cell responses, we next assessed the patterns of $\beta 2 \mathrm{M}, \mathrm{MHC}$ class I, and MHC class II expression on HRS cells. In total, 72 patients had available specimens for analysis of all three antigen presentation components (Data Supplement Table A1B and Fig 3A; representative images in Fig 3B and Data Supplement Fig A6). HRS cell membrane expression of $\beta 2 \mathrm{M}$ was negative in 51 of $72(71 \%)$ tumors, decreased in 16 of $72(22 \%)$, and positive in five of $72(7 \%$; Fig 3A). Similarly, HRS cell membrane expression of MHC class I was negative in 46 of 72 (64\%) patients, decreased in 21 of 72 (29\%), and

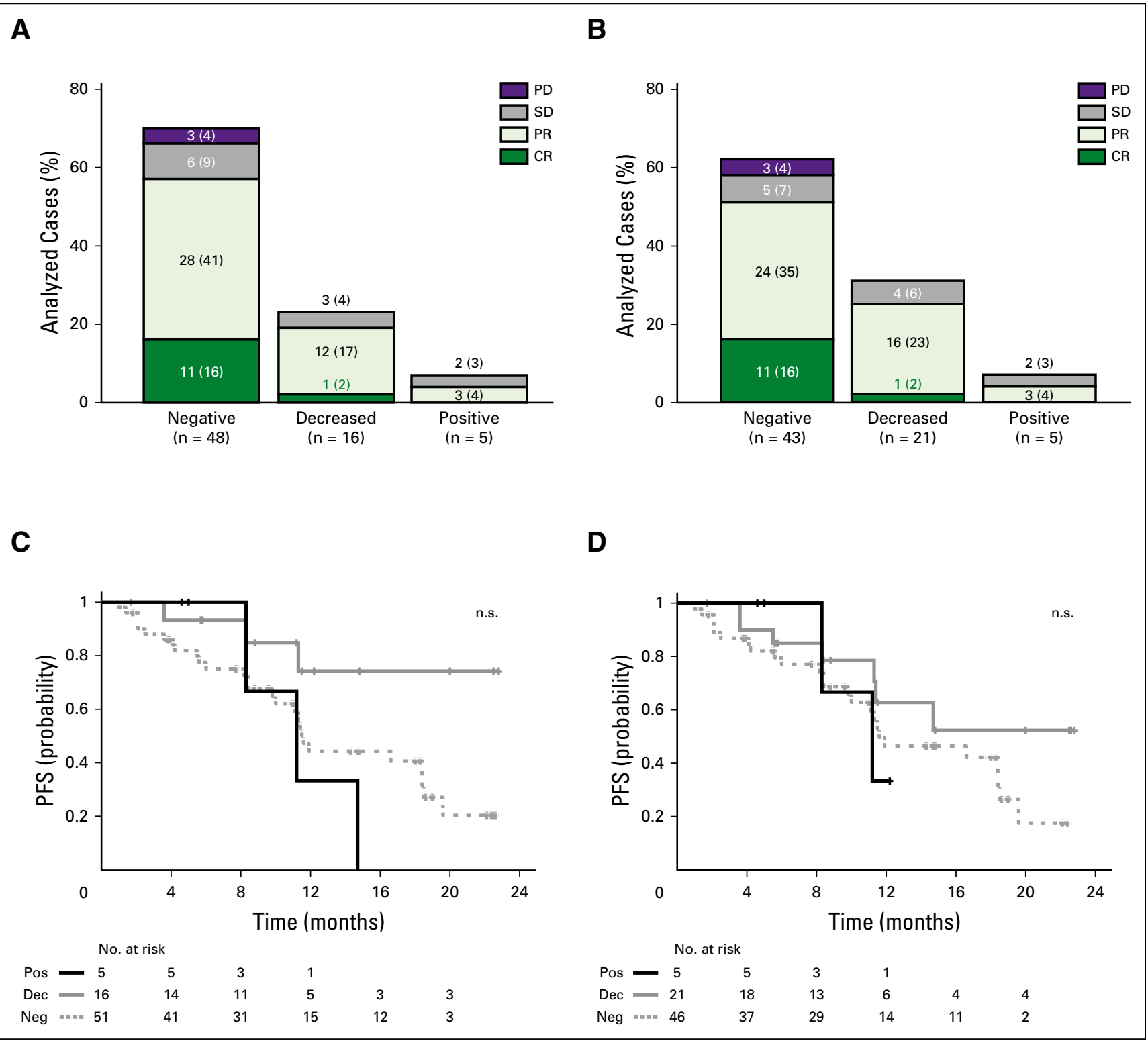

Fig 4. Best overall response (BOR) and progression-free survival (PFS) by $\beta 2$-microglobulin ( $\beta 2 \mathrm{M}$ ) and major histocompatibility complex (MHC) class I expression. Total

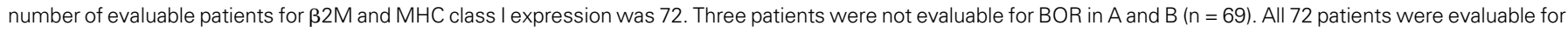

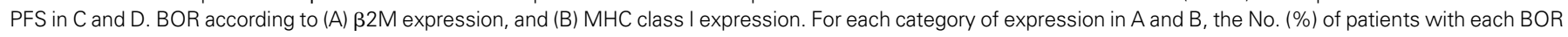

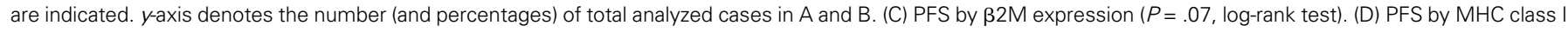

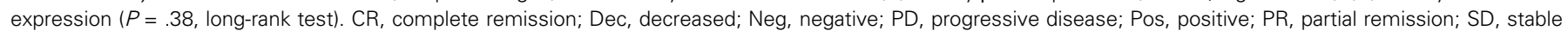
disease. 
positive in five of 72 (7\%; Fig 3A). In this series, there was a highly significant association between HRS cell expression of $\beta 2 \mathrm{M}$ and MHC class I, implicating B2M alterations in the perturbed MHC class I presentation $\left(P<.001\right.$; Data Supplement Table A2). ${ }^{14,15}$ Overall, 93\% (67 of 72) of analyzed cHLs had impaired (negative or decreased) HRS cell surface expression of MHC class I (Fig 3A).

HRS cell membrane expression of MHC class II was negative in 21 of $72(29 \%)$ patients, decreased in 23 of $72(32 \%)$, and positive in 28 of 72 (39\%; Fig 3A). There was no association between the expression of MHC class II and $\beta 2 \mathrm{M}$ or MHC class I on HRS cells $(P=$ .645 and $P=.5764$, respectively; Data Supplement Table A2).

\section{Antigen Presentation and Clinical Outcome}

We next examined the importance of intact MHC class I and II expression on HRS cells for response to PD-1 blockade. First, we assessed the association between BOR, PFS, and $\beta 2 \mathrm{M}$ and $\mathrm{MHC}$ class I expression. Ninety-two percent (11 of 12) of patients who achieved a CR to nivolumab had tumors that were negative for $\beta 2 \mathrm{M}$ and $\mathrm{MHC}$ class I (Figs $4 \mathrm{~A}$ and $4 \mathrm{~B}$ ). There was no association between $\beta 2 \mathrm{M}$ or MHC class I expression on HRS cells and PFS (Figs 4C and 4D). Similar results were obtained in patients with intervals of $\leq 12$ and $>12$ months between ASCT and nivolumab therapy (Data Supplement Fig A7). These data suggest that, in cHL, PD-1 blockade is largely independent of MHC class I-mediated antigen recognition and $\mathrm{CD}^{+}$cytotoxic $\mathrm{T}$-cell responses.

In contrast, $92 \%$ (11 of 12 ) of complete responders had tumors with membranous MHC class II expression on HRS cells (67\% [eight of 12] positive, 25\% [three of 12] decreased; Fig 5A and Data Supplement Fig A8; CR $v$ no CR, $P=.03$ ). MHC class II expression on HRS cells was not predictive for PFS in patients who

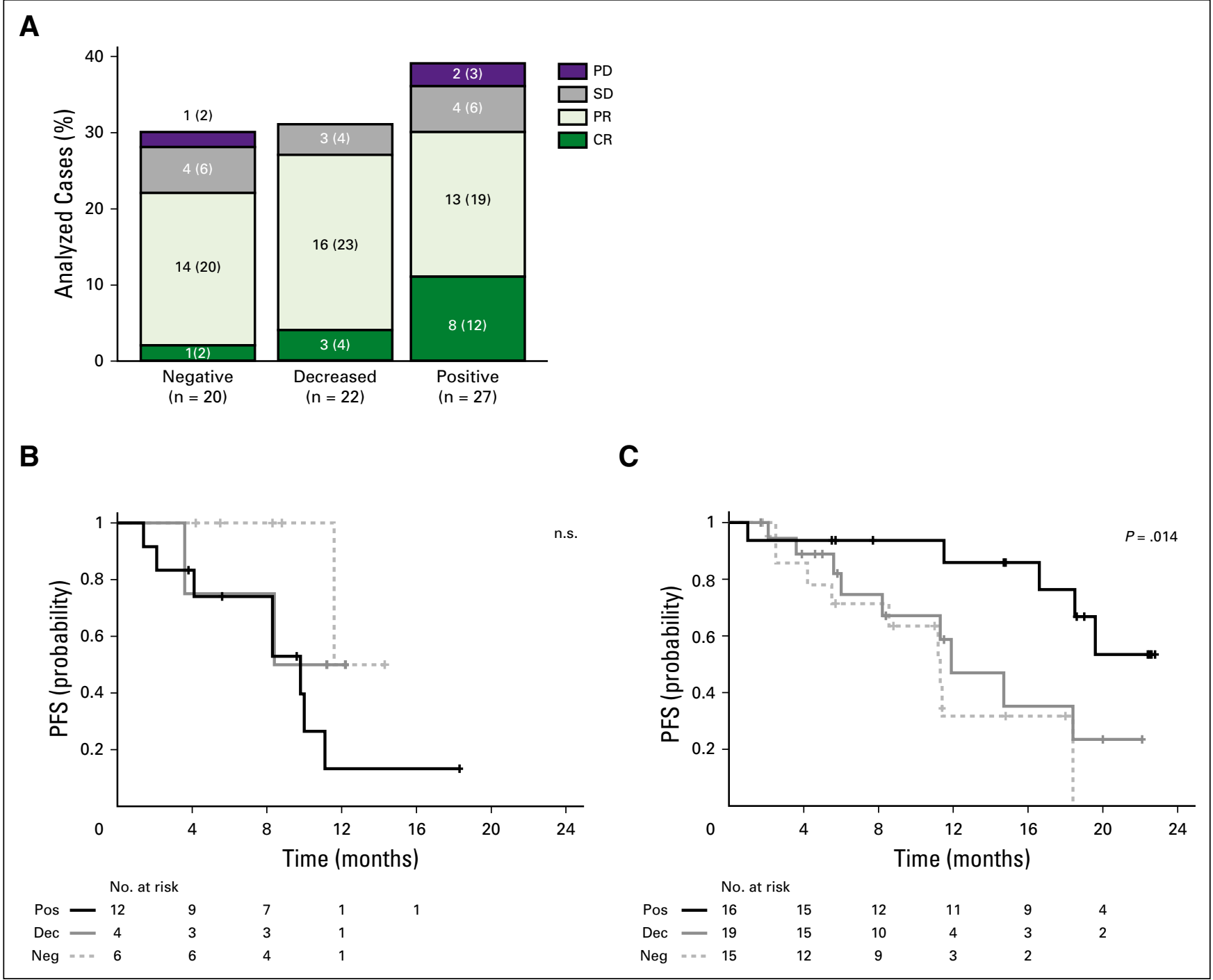

Fig 5. Best overall response (BOR) and progression-free survival (PFS) by major histocompatibility complex (MHC) class II expression. Total number of evaluable patients

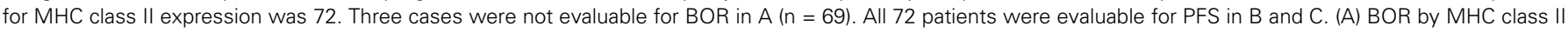

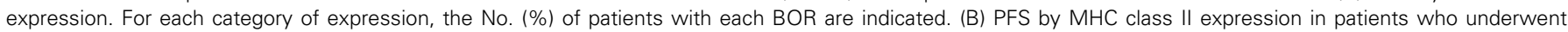

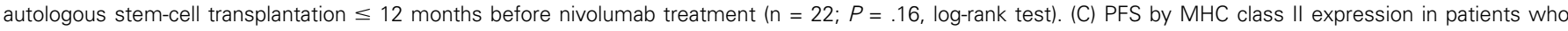

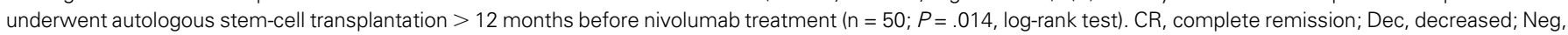
negative; PD, progressive disease; PD-1, programmed death 1; Pos, positive; PR, partial remission; SD, stable disease. 
were treated with nivolumab $\leq 12$ months after myeloablative ASCT, likely because of microenvironmental signals that would not be reflected in archival biopsy specimens (Fig 5B). However, in patients who received the PD-1-blocking antibody $>12$ months after ASCT, positive MHC class II expression on HRS cells was predictive for prolonged PFS (Fig 5C; $P=.014$ ). Taken together, these data highlight a previously unappreciated role of MHC class II-mediated antigen presentation on HRS cells in the clinical response to PD-1 blockade.

\section{DISCUSSION}

In this study of patients with relapsed/refractory cHL, we assessed the possible predictive value of specific immune evasion mechanisms9p24.1/PD-L1/PD-L2 alterations, PD-L1 expression, and perturbed antigen presentation - for response to nivolumab therapy as well as PFS after treatment. All evaluable patients had genetic alterations of 9p24.1/PD-L1/PD-L2 and copy number-dependent increased expression of PD-L1 in HRS cells. Although most patients responded to nivolumab therapy, those with higher-level 9p24.1 alterations and PD-L1 expression on HRS cells had superior PFS. These analyses highlight the importance of quantifying and specifically delineating PD-L1 expression in malignant HRS cells.

Our data suggest that the mechanism of action for PD-1 blockade in cHL may be different from that described in certain solid tumors. ${ }^{10-13}$ In the current study, $93 \%$ of evaluable patients with cHL had absent or decreased expression of $\beta 2 \mathrm{M}$ and MHC class I. In $92 \%$ of complete responders to nivolumab, HRS cells were negative for cell surface $\beta 2 \mathrm{M}$ and MHC class I; moreover, PFS was unrelated to HRS cell expression of $\beta 2 \mathrm{M}$ or MHC class I. These findings strongly suggest that $\beta 2 \mathrm{M}$ deficiency is a major mechanism of MHC class I loss in cHL, and PD-1 blockade is largely independent of MHC class I-mediated antigen recognition and associated $\mathrm{CD}^{+}$cytotoxic T-cell responses in cHL.

In marked contrast, $92 \%$ of complete responders to nivolumab had membranous MHC class II expression on their HRS cells. The observed variability in MHC class II expression on HRS cells may have genetic bases, such as inactivating rearrangements of the MHC class II transactivator CIITA ${ }^{19}$ and downregulation of CIITA by Epstein-Barr virus latent membrane protein $2 \mathrm{~A}^{20}$ In patients with fully reconstituted immune systems ( $>12$ months from myeloablative ASCT), positive MHC class II expression on HRS cells was also predictive for prolonged PFS. Of note, a subset of patients with MHC class II-negative HRS cells also had short-lived responses to nivolumab, suggesting that additional mechanisms remain to be defined.
These initial analyses were limited by the availability of tumor biopsy specimens from patients in the CheckMate 205 trial and should be prospectively validated in additional trials of PD-1 blockade in relapsed/refractory cHL. ${ }^{9}$ However, our data already prompt speculation regarding an alternative $\mathrm{CD} 4{ }^{+} \mathrm{T}$ cell-mediated mechanism of response to PD-1 blockade in cHL and suggest associated biomarkers of response and resistance and additional complementary targets, such as the MHC class II-interacting checkpoint protein, LAG3. ${ }^{28}$

Despite the focus on $\mathrm{CD}^{+} \mathrm{T}$ cell-mediated mechanisms of PD-1 blockade, ${ }^{10-13}$ recent studies also highlight the role of MHC class II-associated antigen presentation and $\mathrm{CD} 4^{+}$-infiltrating $\mathrm{T}$ cells in certain solid tumors ${ }^{16}$ and define tumor neoantigens that are largely recognized by $\mathrm{CD} 4^{+} \mathrm{T}$ cells. ${ }^{17,18,29}$ Taken together, these findings support the hypothesis of an alternative MHC class IIdependent, $\mathrm{CD}^{+}{ }^{+} \mathrm{T}$ cell-associated mechanism of action of PD-1 blockade in cHL and, possibly, other tumors.

\section{AUTHORS' DISCLOSURES OF POTENTIAL CONFLICTS OF INTEREST}

Disclosures provided by the authors are available with this article at jco.org.

\section{AUTHOR CONTRIBUTIONS}

Conception and design: Margaretha G.M. Roemer, Scott J. Rodig, Margaret A. Shipp

Provision of study materials or patients: Jing Ouyang, Stephanie Sasse, Anas Younes, Michelle Fanale, Armando Santoro, Pier Luigi Zinzani, John Timmerman, Graham P. Collins, Radhakrishnan Ramchandren, Jonathon B. Cohen, Jan Paul de Boer, John Kuruvilla, Kerry J. Savage, Marek Trneny, Stephen Ansell, Kazunobu Kato, Benedetto Farsaci, Anne Sumbul, Philippe Armand

Collection and assembly of data: Margaretha G.M. Roemer, Robert A. Redd, Fathima Zumla Cader, Christine J. Pak, Sara Abdelrahman, Donna S. Neuberg, Geraldine S. Pinkus, Azra H. Ligon, Scott J. Rodig, Margaret A. Shipp

Data analysis and interpretation: Margaretha G.M. Roemer, Robert A. Redd, Fathima Zumla Cader, Jing Ouyang, Stephanie Sasse, Anas Younes, Michelle Fanale, Armando Santoro, Pier Luigi Zinzani, John Timmerman, Graham P. Collins, Radhakrishnan Ramchandren, Jonathon B. Cohen, Jan Paul de Boer, John Kuruvilla, Kerry J. Savage, Marek Trneny, Stephen Ansell, Kazunobu Kato, Benedetto Farsaci, Anne Sumbul, Philippe Armand, Donna S. Neuberg, Geraldine S. Pinkus, Azra H. Ligon, Scott J. Rodig, Margaret A. Shipp

Manuscript writing: All authors

Final approval of manuscript: All authors

Accountable for all aspects of the work: All authors

\section{REFERENCES}

1. Mathas $S$, Hartmann S, Küppers R: Hodgkin lymphoma: Pathology and biology. Semin Hematol 53:139-147, 2016

2. Juszczynski $P$, Ouyang J, Monti S, et al: The AP1-dependent secretion of galectin-1 by Reed Sternberg cells fosters immune privilege in classical Hodgkin lymphoma. Proc Natl Acad Sci USA 104: 13134-13139, 2007
3. Green MR, Monti S, Rodig SJ, et al: Integrative analysis reveals selective 9p24.1 amplification, increased PD-1 ligand expression, and further induction via JAK2 in nodular sclerosing Hodgkin lymphoma and primary mediastinal large B-cell lymphoma. Blood 116:3268-3277, 2010

4. Roemer MG, Advani $\mathrm{RH}$, Ligon $\mathrm{AH}$, et al: $\mathrm{PD}$ L1 and PD-L2 genetic alterations define classical Hodgkin lymphoma and predict outcome. J Clin Oncol 34:2690-2697, 2016
5. Baumeister SH, Freeman GJ, Dranoff G, et al: Coinhibitory pathways in immunotherapy for cancer. Annu Rev Immunol 34:539-573, 2016

6. Ansell SM, Lesokhin AM, Borrello I, et al: PD-1 blockade with nivolumab in relapsed or refractory Hodgkin's lymphoma. N Engl J Med 372:311-319, 2015

7. Younes A, Santoro A, Shipp M, et al: Nivolumab for classical Hodgkin's lymphoma after failure of both autologous stem-cell transplantation and brentuximab vedotin: A multicentre, multicohort, singlearm phase 2 trial. Lancet Oncol 17:1283-1294, 2016 
8. Armand $P$, Shipp MA, Ribrag $V$, et al: Programmed death-1 blockade with pembrolizumab in patients with classical Hodgkin lymphoma after brentuximab vedotin failure. J Clin Oncol 34:3733-3739 2016

9. Chen R, Zinzani PL, Fanale MA, et al: Phase II study of the efficacy and safety of pembrolizumab for relapsed/refractory classic Hodgkin lymphoma. J Clin Oncol 35:2125-2132, 2017

10. Tumeh PC, Harview CL, Yearley JH, et al: PD-1 blockade induces responses by inhibiting adaptive immune resistance. Nature 515:568-571, 2014

11. Im SJ, Hashimoto $M$, Gerner MY, et al: Defining CD8+ T cells that provide the proliferative burst after PD-1 therapy. Nature 537:417-421, 2016

12. Kamphorst $A O$, Wieland $A$, Nasti $T$, et al: Rescue of exhausted CD8 T cells by PD-1-targeted therapies is CD28 dependent. Science 355:1423-1427, 2017

13. Juneja VR, McGuire KA, Manguso RT, et al: PD-L1 on tumor cells is sufficient for immune evasion in immunogenic tumors and inhibits CD8 T cell cytotoxicity. J Exp Med 214:895-904, 2017

14. Reichel J, Chadburn A, Rubinstein PG, et al: Flow sorting and exome sequencing reveal the oncogenome of primary Hodgkin and ReedSternberg cells. Blood 125:1061-1072, 2015

15. Roemer MG, Advani $\mathrm{RH}$, Redd RA, et al: Classical Hodgkin lymphoma with reduced $\beta 2 \mathrm{M}$ / $\mathrm{MHC}$ class I expression is associated with inferior outcome independent of 9p24.1 status. Cancer Immunol Res 4:910-916, 2016

16. Johnson DB, Estrada MV, Salgado $R$, et al: Melanoma-specific MHC-II expression represents a tumour-autonomous phenotype and predicts response to anti-PD-1/PD-L1 therapy. Nat Commun 7: 10582, 2016

17. Kreiter S, Vormehr M, van de Roemer N, et al: Mutant MHC class II epitopes drive therapeutic immune responses to cancer. Nature 520:692-696, 2015 [Erratum: Nature 523:370, 2015]

18. Linnemann $C$, van Buuren $M M$, Bies $L$, et al: High-throughput epitope discovery reveals frequent recognition of neo-antigens by $\mathrm{CD} 4+\mathrm{T}$ cells in human melanoma. Nat Med 21:81-85, 2015

19. Steidl C, Shah SP, Woolcock BW, et al: MHC class ॥ transactivator CIITA is a recurrent gene fusion partner in lymphoid cancers. Nature 471:377-381, 2011

20. Lin JH, Lin JY, Chou YC, et al: Epstein-Barr virus LMP2A suppresses MHC class II expression by regulating the $B$-cell transcription factors E47 and PU.1. Blood 125:2228-2238, 2015

21. Diepstra $A$, van Imhoff GW, Karim-Kos HE, et al: HLA class II expression by Hodgkin Reed-Sternberg cells is an independent prognostic factor in classical Hodgkin's lymphoma. J Clin Oncol 25:3101-3108, 2007

22. Carey $C D$, Gusenleitner D, Lipschitz $M$, et al: Topological analysis reveals a PD-L1-associated microenvironmental niche for Reed-Sternberg cells in Hodgkin lymphoma. Blood 130:2420-2430, 2017

23. Cheson BD, Pfistner $B$, Juweid $M E$, et al: Revised response criteria for malignant lymphoma. J Clin Oncol 25:579-586, 2007

24. Mahoney KM, Sun $H$, Liao $X$, et al: PD-L1 antibodies to its cytoplasmic domain most clearly delineate cell membranes in immunohistochemical staining of tumor cells. Cancer Immunol Res 3: 1308-1315, 2015

25. Mackall CL: T-cell immunodeficiency following cytotoxic antineoplastic therapy: A review. Stem Cells 18:10-18, 2000

26. Głowala-Kosińska M, Chwieduk A, Smagur A et al: Thymic activity and $T$ cell repertoire recovery after autologous hematopoietic stem cell transplantation preceded by myeloablative radiotherapy or chemotherapy. Biol Blood Marrow Transplant 22: 834-842, 2016

27. Ljungman $P$, Cordonnier $C$, Einsele $H$, et al: Vaccination of hematopoietic cell transplant recipients. Bone Marrow Transplant 44:521-526, 2009

28. Andrews LP, Marciscano AE, Drake CG, et al: LAG3 (CD223) as a cancer immunotherapy target. Immunol Rev 276:80-96, 2017

29. Ott PA, Hu Z, Keskin DB, et al: An immunogenic personal neoantigen vaccine for patients with melanoma. Nature 547:217-221, 2017

\section{Affiliations}

Margaretha G.M. Roemer, Robert A. Redd, Fathima Zumla Cader, Christine J. Pak, Sara Abdelrahman, Jing Ouyang, Philippe Armand, Donna S. Neuberg, and Margaret A. Shipp, Dana-Farber Cancer Institute; Geraldine S. Pinkus, Azra H. Ligon, and Scott J. Rodig, Brigham and Women's Hospital, Boston, MA; Margaretha G.M. Roemer, VU University Medical Center; Jan Paul De Boer, Antoni van Leeuwenhoek Hospital, Lunenburg Phase I/II Consortium, Amsterdam, the Netherlands; Stephanie Sasse, University Hospital of Cologne, Cologne, Germany; Anas Younes, Memorial Sloan Kettering Cancer Center, New York, NY; Michelle Fanale, University of Texas MD Anderson Cancer Center, Houston, TX; Armando Santoro, Humanitas University, Rozzano, Milan; Pier Luigi Zinzani, University of Bologna, Bologna, Italy; John Timmerman, University of California, Los Angeles Medical Center, Los Angeles, CA; Graham P. Collins, Churchill Hospital, Oxford, United Kingdom; Radhakrishnan Ramchandren, Barbara Ann Karmanos Cancer Institute, Detroit, MI; Jonathon B. Cohen, Emory University, Atlanta, GA; John Kuruvilla, Princess Margaret Cancer Centre, Toronto, Ontario, Canada; Kerry J. Savage, British Columbia Cancer Agency Center for Lymphoid Cancer, Vancouver, British Columbia, Canada; Marek Trneny, Charles University in Prague, General University Hospital in Prague, Prague, Czech Republic; Stephen Ansell, Mayo Clinic, Rochester, MN; and Kazunobu Kato, Benedetto Farsaci, and Anne Sumbul, Bristol-Myers Squibb, Princeton, NJ.

\section{Support}

Funded by National Institutes of Health Grants No. NIH R01CA061026 (M.A.S.) and NIH 5P30 CA006516 (R.A.R.), the Miller Family Fund (M.A.S.), and Bristol-Myers Squibb (S.J.R. and M.A.S.). 


\section{AUTHORS' DISCLOSURES OF POTENTIAL CONFLICTS OF INTEREST}

Major Histocompatibility Complex Class II and Programmed Death Ligand 1 Expression Predict Outcome After Programmed Death 1 Blockade in Classic Hodgkin Lymphoma

The following represents disclosure information provided by authors of this manuscript. All relationships are considered compensated. Relationships are self-held unless noted. I = Immediate Family Member, Inst = My Institution. Relationships may not relate to the subject matter of this manuscript. For more information about ASCO's conflict of interest policy, please refer to www.asco.org/rwc or ascopubs.org/jco/site/ifc.

Margaretha G.M. Roemer

No relationship to disclose

\section{Robert A. Redd}

No relationship to disclose

Fathima Zumla Cader

No relationship to disclose

Christine J. Pak

No relationship to disclose

\section{Sara Abdelrahman}

No relationship to disclose

Jing Ouyang

No relationship to disclose

\section{Stephanie Sasse}

No relationship to disclose

\section{Anas Younes}

Honoraria: Bristol-Myers Squibb, Bayer HealthCare Pharmaceuticals, Celgene, Incyte, Janssen Pharmaceuticals, Merck, Sanofi, Seattle Genetics, Takeda Millennium

Research Funding: Curis (Inst), Johnson \& Johnson (Inst), Novartis (Inst)

\section{Michelle Fanale}

Honoraria: Seattle Genetics, Takeda, Research to Practice, Plexus, BristolMyers Squibb

Consulting or Advisory Role: Spectrum Pharmaceuticals, Amgen, Merck, Bristol-Myers Squibb, Seattle Genetics

Research Funding: Millennium, Seattle Genetics, Novartis, MedImmune, Celgene, Molecular Templates, Genentech, Gilead Sciences, Bristol-Myers Squibb, Merck, ADC Therapeutics, Pharmacyclics, Takeda

Travel, Accommodations, Expenses: Takeda, Spectrum Pharmaceuticals, Research to Practice, Plexus, Bristol-Myers Squibb, Merck, Seattle Genetics

\section{Armando Santoro}

Consulting or Advisory Role: Takeda, Eli Lilly, Amgen, Bayer HealthCare Pharmaceuticals, ArQule

Pier Luigi Zinzani

Consulting or Advisory Role: Bayer HealthCare Pharmaceuticals, BristolMyers Squibb, Celgene, Gilead, Johnson \& Johnson, Karyopharm Therapeutics, Merck, Roche, Servier, Takeda, Verastem

\section{John Timmerman}

Stock or Other Ownership: Biomarin Pharmaceutical, Medivation

Honoraria: Bristol-Myers Squibb

Consulting or Advisory Role: Celgene, Seattle Genetics, Tenobio Research Funding: Bristol-Myers Squibb, ImmunGene, Kite Pharma Travel, Accommodations, Expenses: Bristol-Myers Squibb

\section{Graham P. Collins}

Honoraria: Gilead, Roche, Takeda

Consulting or Advisory Role: Gilead, Roche, Takeda, Bristol-Myers Squibb, Pfizer, MSD, Celleron Therapeutics

Speakers' Bureau: Gilead, Takeda, Roche

Research Funding: Celgene (Inst), MSD (Inst), Amgen (Inst)

Travel, Accommodations, Expenses: Napp Pharmaceuticals, Roche, Takeda
Radhakrishnan Ramchandren

Research Funding: Janssen Pharmaceuticals, (Inst), Merck (Inst), Pharmacyclics (Inst), Seattle Genetics (Inst)

Jonathon B. Cohen

Consulting or Advisory Role: AbbVie, Celgene, Infinity Pharmaceuticals, Millennium, Novartis

Research Funding: Bristol-Myers Squibb (Inst), Novartis (Inst), Takeda (Inst)

Jan Paul de Boer

Consulting or Advisory Role: Bristol-Myers Squibb, Merck, MDS, Eisai Research Funding: Merck (Inst)

John Kuruvilla

Honoraria: Bristol-Myers Squibb, Amgen, Celgene, Gilead, Roche, Janssen Pharmaceuticals, Lundbeck, Merck, Seattle Genetics

Consulting or Advisory Role: Bristol-Myers Squibb, Gilead, Janssen Pharmaceuticals, Hoffman-La Roche, Merck, Seattle Genetics, Lymphoma Canada

Research Funding: Leukemia and Lymphoma Society US (Inst), Jesse \& Julie Rasch Foundation (Inst), Celgene (Inst), Roche (Inst)

Kerry J. Savage

Honoraria: Bristol-Myers Squibb, Seattle Genetics, Merck, Gilead, Takeda, Novartis

Consulting or Advisory Role: Bristol-Myers Squibb, Seattle Genetics, Merck, Infinity, Gilead, SERVIER

\section{Marek Trneny}

Honoraria: Genentech, Celgene, Incyte, Janssen Pharmaceuticals, Gilead Sciences, Takeda, Bristol-Myers Squibb, TG Therapeutics

Consulting or Advisory Role: Genentech, Celgene, Gilead Sciences, Takeda, Bristol-Myers Squibb, Incyte, Janssen Pharmaceuticals, TG Therapeutics

Travel, Accommodations, Expenses: Gilead Sciences, Takeda, BristolMyers Squibb, Roche

Stephen Ansell

Honoraria: WebMD, Research to Practice

Research Funding: Bristol-Myers Squibb (Inst), Celldex Therapeutics (Inst), Seattle Genetics (Inst), Merck (Inst), Affimed (Inst), Trillium Therapeutics (Inst)

\section{Kazunobu Kato}

Employment: Bristol-Myers Squibb

Stock or Other Ownership: Bristol-Myers Squibb

Benedetto Farsaci

Employment: Bristol-Myers Squibb

Anne Sumbul

Employment: Bristol-Myers Squibb

Philippe Armand

Consulting or Advisory Role: Bristol-Myers Squibb, Merck, Infinity, Pfizer

Research Funding: Bristol-Myers Squibb (Inst), Affimed (Inst), Merck (Inst), Otsuka Pharmaceutical (Inst), Pfizer (Inst), Roche (Inst), Sequenta (Inst), Sigma-Tau (Inst), Tensha Therapeutics (Inst) 
Donna S. Neuberg

Stock or Other Ownership: Madrigal Pharmaceuticals

Patents, Royalties, Other Intellectual Property: Two patents for mutation interpretation in patients with myelodysplastic syndromes: PCT/US2012/ 042734, PCT/US2014/037717

\section{Geraldine S. Pinkus}

No relationship to disclose

\section{Azra H. Ligon}

No relationship to disclose
Scott J. Rodig

Research Funding: Bristol-Myers Squibb (Inst)

Travel, Accommodations, Expenses: Bristol-Myers Squibb

\section{Margaret A. Shipp}

Honoraria: Bristol-Myers Squibb, AstraZeneca

Consulting or Advisory Role: Bristol-Myers Squibb

Research Funding: Bristol-Myers Squibb (Inst), Bayer HealthCare Pharmaceuticals, (Inst) 
MHC Class II, PD-L1, and Outcome After PD-1 Blockade

\section{Acknowledgment}

Editorial assistance was provided by Caudex, funded by Bristol-Myers Squibb. 